\title{
Inverse Dynamics-based Adaptive Control of Nonlinear Bilateral Teleoperation Systems
}

\author{
X. Liu and M. Tavakoli
}

\begin{abstract}
Inverse dynamics controllers deal with nonlinear terms in the robot dynamics in a way that, in the ideal case, the closed-loop system becomes linear and decoupled. Consequently, the performance of the closed-loop systems will be easy to study. Due to such an advantage, inverse dynamics-based adaptive control has been applied to motion control of an uncertain robot in free motion in the literature. However, so far there has been no attempt at simultaneous motion and force control in a master-slave haptic teleoperation system using an adaptive inverse dynamics approach. In this paper, for multi-degree-of-freedom teleoperation systems with nonlinear and uncertain dynamics, adaptive inverse dynamics controllers are incorporated into Lawrence's 4-channel bilateral teleoperation control framework. The resulting high-fidelity control system does not need exact knowledge of the dynamics of the master or the slave. A Lyapunov function is presented to analyze the transparency of the teleoperation system. A simulation study is included to demonstrate the effectiveness of the proposed control method.
\end{abstract}

\section{INTRODUCTION}

$\mathrm{I}^{\mathrm{n}}$ a master-slave teleoperation system, the human operator applies a force on the master to control the position of the slave and perform a task in the remote environment. If the slave exactly reproduces the master's position and the master accurately reproduces the slave-environment contact force, the teleoperation system is said to be fully transparent [1]. In terms of ensuring full transparency, Lawrence's 4-channel control architecture is the most successful among other methods [2]. A limitation of the conventional 4-channel controller is that it assumes perfect knowledge of the master and the slave linear impedances. Nevertheless, the exact knowledge of the master and the slave dynamics may be unavailable due to model uncertainties. Thus, in recent years adaptive control has been employed to compensate for the parametric dynamic uncertainties in teleoperation systems.

There are a few adaptive control schemes for linear master and slave models where the slave and the environment dynamics are allowed to be uncertain [3], [4]. In both of these, the dynamics of the master and the slave were assumed to be linear. In addition, an adaptive controller was only designed

This research was supported by the Natural Sciences and Engineering Research Council (NSERC) of Canada under grant RGPIN-372042 and by the China Scholarship Council (CSC) under grant [2009]3012.

$\mathrm{X}$. Liu is with the School of Automation Engineering, University of Electronic Science and Technology of China, Chengdu, Sichuan, 611731 China. She is now a visiting PhD student in the Department of Electrical and Computer Engineering, University of Alberta, Edmonton, Alberta, T6G 2V4 Canada. (e-mail: xia8@ualberta.ca).

M. Tavakoli is with the Department of Electrical and Computer Engineering, University of Alberta, Edmonton, Alberta, T6G 2V4 Canada. (e-mail: tavakoli@ece.ualberta.ca). for the slave, and a fixed compensator was used for the master.

As multi-DOF robot dynamics are generally nonlinear, adaptive control schemes for nonlinear and uncertain master and slave models have been developed in [5]-[8] where adaptive controllers were used for both the master and the slave. Adaptive controllers are proposed to ensure state synchronization for positions and velocities of the master and slave robots in [9], [10]. However, the limitation of both of them is that the adaptive controllers are designed for master/slave robots in free motion but not in contact motion. Furthermore, none of the above adaptive control schemes has taken advantage of the inverse dynamics approach, which has the advantage of providing a nonlinear feedback control law canceling the nonlinear terms in the closed-loop dynamics if the dynamics were perfectly known.

Adaptive inverse dynamics control of the position of a robot under dynamic uncertainties was investigated in [11]-[13]. However, the results in [11]-[13] have only been applied to motion control of a single robot in free motion. So far, simultaneous motion and force control in a master-slave teleoperation system, in which the master and the slave are allowed to make contact with the human operator and the environment, has not been addressed.

This paper proposes adaptive bilateral controllers based on the inverse dynamics approach for teleoperation systems with nonlinear and uncertain dynamics. The rest of this paper is organized as follows. In Section II, the 4-channel control architecture is modified to encompass adaptive inverse dynamics position controllers for the master and slave robots. In Section III, the adaptive controllers are presented and a unified closed-loop dynamics for the overall master-slave system is found. In Section IV, a Lyapunov function is constructed and position and force tracking performance of the overall teleoperation system is investigated. In Section V, simulations are done to demonstrate the effectiveness of the proposed controller. The paper is concluded in Section VI.

\section{ARCHITECTURE OF ADAPTIVE INVERSE DYNAMICS 4-CHANNEL TELEOPERATION CONTROL}

The 4-channel architecture in Fig. 1 has a position controller comprised of $\mathrm{C}_{\mathrm{L}}$ and $\mathrm{C}_{4}$ for the master, and another position controller comprised of $\mathrm{C}_{\mathrm{R}}$ and $\mathrm{C}_{1}$ for the slave. The controllers $\mathrm{C}_{2}$ and $\mathrm{C}_{3}$ are feedback force terms for the master and the slave, respectively. Also, $\mathrm{C}_{6}$ and $\mathrm{C}_{5}$ are local force feedforward controller for the master and for the slave, respectively. The signals $\mathbf{f}_{\mathrm{h}}^{*}$ and $\mathbf{f}_{\mathrm{e}}^{*}$ denote the exogenous forces of the operator and the environment, respectively. The rest of variables in Fig. 1 will be introduced 
in Section III, and we merely describe the general architecture of the proposed scheme in this section.

The basic idea of inverse dynamics control is to seek a nonlinear feedback law to cancel the nonlinear terms in a dynamic model. Our proposed teleoperation control scheme is based on incorporating two adaptive inverse dynamics position controllers for the master and the slave into the 4-channel architecture. As it can be seen in Fig. 1, the position controller comprising $\mathrm{C}_{\mathrm{L}}$ and $\mathrm{C}_{4}$ for the master and $\mathrm{C}_{\mathrm{R}}$ and
$\mathrm{C}_{1}$ for the slave are replaced by two designed adaptive inverse dynamics position controllers. The block diagrams of the inverse dynamics position controllers for the master and the slave, to be designed in the Section III, are shown in Fig. 2. Note that the remaining control blocks in Fig. 1, i.e., $\mathrm{C}_{2}, \mathrm{C}_{3}$, $\mathrm{C}_{5}$ and $\mathrm{C}_{6}$ (where $\mathrm{C}_{5}=\mathrm{C}_{3}-1$ and $\mathrm{C}_{6}=\mathrm{C}_{2}-1$ for transparency reasons) continue to be used as force feedback and feedforward controllers in the proposed approach.

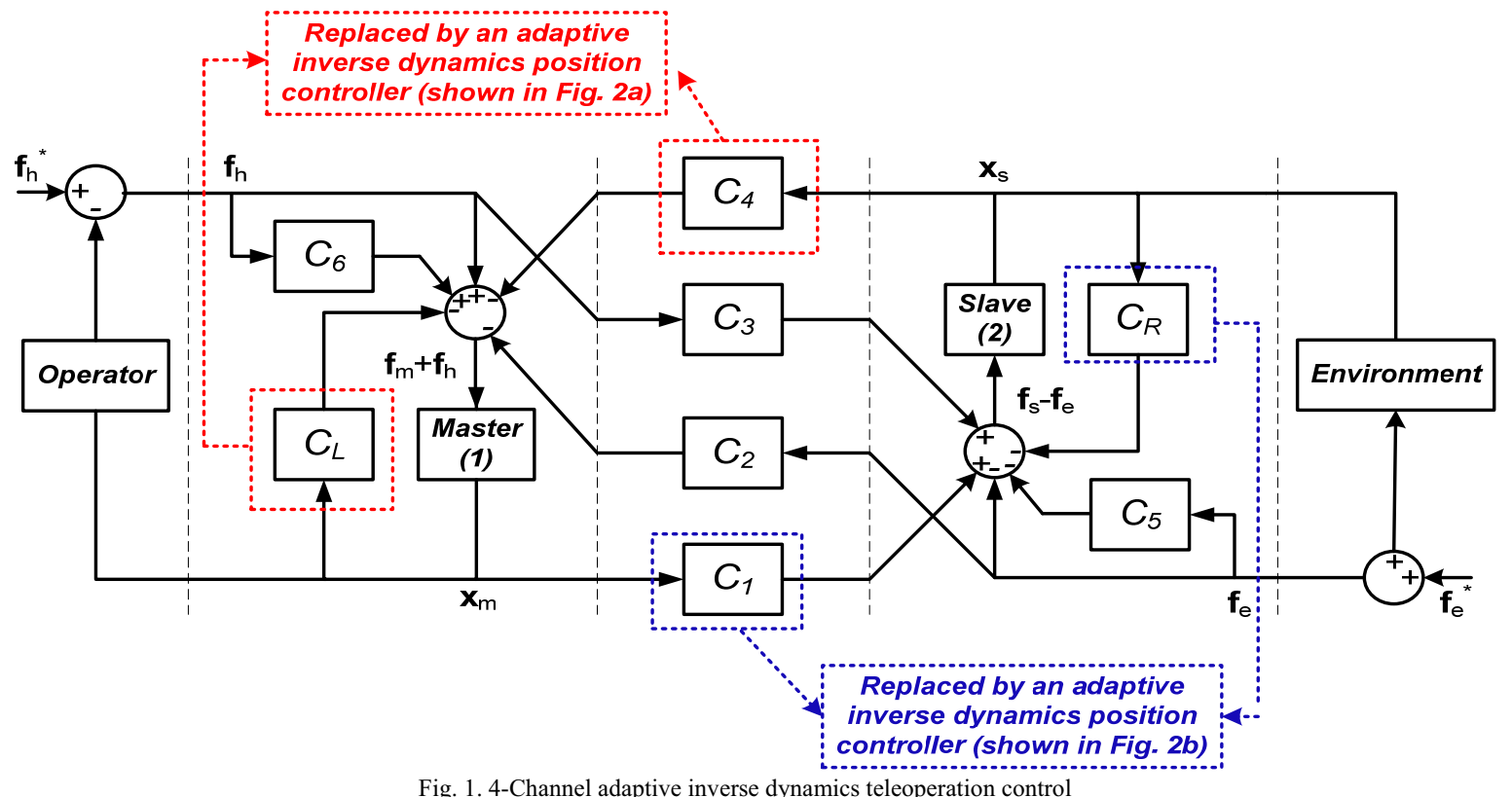

Fig. 1. 4-Channel adaptive inverse dynamics teleoperation control

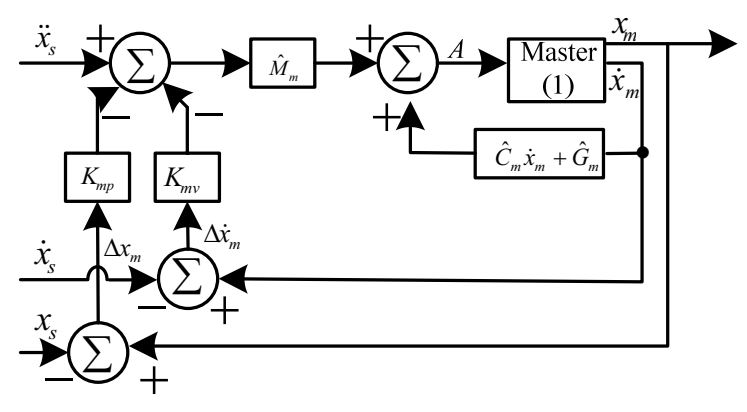

$\mathbf{A}=\widehat{\mathbf{M}}_{\mathrm{m}}\left(\ddot{\mathbf{x}}_{\mathrm{s}}-\mathrm{K}_{\mathrm{mv}} \Delta \dot{\mathbf{x}}_{\mathrm{m}}-\mathrm{K}_{\mathrm{mp}} \Delta \mathbf{x}_{\mathrm{m}}\right)+\widehat{\mathbf{C}}_{\mathrm{m}} \dot{\mathbf{x}}_{\mathrm{m}}+\widehat{\mathbf{G}}_{\mathrm{m}}$

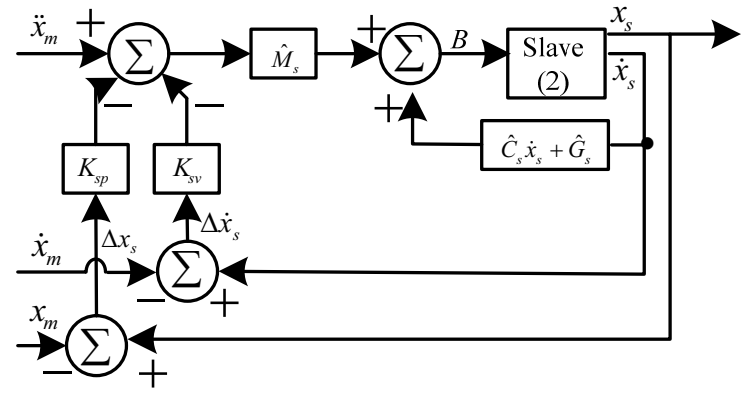

$\mathbf{B}=\widehat{\mathbf{M}}_{\mathrm{s}}\left(\ddot{\mathbf{x}}_{\mathrm{m}}-\mathrm{K}_{\mathrm{sv}} \Delta \dot{\mathbf{x}}_{\mathrm{s}}-\mathrm{K}_{\mathrm{sp}} \Delta \mathbf{x}_{\mathrm{s}}\right)+\widehat{\mathbf{C}}_{\mathrm{s}} \dot{\mathbf{x}}_{\mathrm{s}}+\widehat{\mathbf{G}}_{\mathrm{s}}$

Fig. 2. Adaptive inverse dynamics position controllers (a) for the master (left), and (b) for the slave (right)

\section{MODELS AND CONTROLLERS}

\section{A. Model of Nonlinear Teleoperation System}

When interacting with a human and an environment, the task-space nonlinear dynamic models for $n$-DOF master and slave robots can be written as

$\mathbf{M}_{\mathrm{m}}\left(\mathbf{q}_{\mathrm{m}}\right) \ddot{\mathbf{x}}_{\mathrm{m}}+\mathbf{C}_{\mathrm{m}}\left(\mathbf{q}_{\mathrm{m}}, \dot{\mathbf{q}}_{\mathrm{m}}\right) \dot{\mathbf{x}}_{\mathrm{m}}+\mathbf{G}_{\mathrm{m}}\left(\mathbf{q}_{\mathrm{m}}\right)=\mathbf{f}_{\mathrm{m}}+\mathbf{f}_{\mathrm{h}}$

$$
\mathbf{M}_{\mathrm{s}}\left(\mathbf{q}_{\mathrm{s}}\right) \ddot{\mathbf{x}}_{\mathrm{s}}+\mathbf{C}_{\mathrm{s}}\left(\mathbf{q}_{\mathrm{s}}, \dot{\mathbf{q}}_{\mathrm{s}}\right) \dot{\mathbf{x}}_{\mathrm{s}}+\mathbf{G}_{\mathrm{s}}\left(\mathbf{q}_{\mathrm{s}}\right)=\mathbf{f}_{\mathrm{s}}-\mathbf{f}_{\mathrm{e}}
$$

where $\mathbf{x}_{\mathrm{m}}, \mathbf{x}_{\mathrm{s}} \in \mathrm{R}^{6 \times 1}$ are end-effector Cartesian positions (and orientations), $\mathrm{q}_{\mathrm{m}}, \mathrm{q}_{\mathrm{s}} \in \mathrm{R}^{\mathrm{n} \times 1}$ are joint angle positions, $\mathbf{M}_{\mathrm{m}}\left(\mathbf{q}_{\mathrm{m}}\right), \mathbf{M}_{\mathrm{s}}\left(\mathbf{q}_{\mathrm{s}}\right) \in \mathrm{R}^{6 \times 6}$ are symmetric positive-definite inertia matrices, $\mathbf{C}_{\mathrm{m}}\left(\mathbf{q}_{\mathrm{m}}, \dot{\mathbf{q}}_{\mathrm{m}}\right), \mathbf{C}_{\mathrm{s}}\left(\mathbf{q}_{\mathrm{s}}, \dot{\mathbf{q}}_{\mathrm{s}}\right) \in \mathrm{R}^{6 \times 6}$ are Coriolis and centrifugal terms, and $\mathbf{G}_{\mathrm{m}}\left(\mathbf{q}_{\mathrm{m}}\right), \mathbf{G}_{\mathrm{s}}\left(\mathbf{q}_{\mathrm{s}}\right) \in \mathrm{R}^{6 \times 1}$ are gravity terms for the master and the slave, respectively. Also, $\mathbf{f}_{\mathrm{m}}, \mathbf{f}_{\mathrm{s}} \in \mathrm{R}^{6 \times 1}$ are force/torque control signals for the 
master and the slave, $\mathbf{f}_{\mathrm{h}} \in \mathrm{R}^{6 \times 1}$ is the force (and torque) that the operator applies to the master, and $\mathbf{f}_{\mathrm{e}} \in \mathrm{R}^{6 \times 1}$ is the force (and torque) that the environment applies to the slave.

Property 1. The left sides of (1) and (2) are linear in a set of dynamic parameters $\boldsymbol{\theta}_{m d}=\left(\theta_{\mathrm{md} 1}, \ldots, \theta_{\mathrm{mdp}_{1}}\right)^{\mathrm{T}}$ and $\boldsymbol{\theta}_{\mathrm{sd}}=$ $\left(\theta_{\mathrm{sd} 1}, \ldots, \theta_{\mathrm{sdp}_{2}}\right)^{\mathrm{T}}$ as [14]:

$$
\begin{aligned}
& \mathbf{M}_{\mathrm{m}}\left(\mathbf{q}_{\mathrm{m}}\right) \ddot{\mathbf{x}}_{\mathrm{m}}+\mathbf{C}_{\mathrm{m}}\left(\mathbf{q}_{\mathrm{m}}, \dot{\mathbf{q}}_{\mathrm{m}}\right) \dot{\mathbf{x}}_{\mathrm{m}}+\mathbf{G}_{\mathrm{m}}\left(\mathbf{q}_{\mathrm{m}}\right) \\
& \quad=\mathbf{Y}_{\mathrm{md}}\left(\mathbf{q}_{\mathrm{m}}, \dot{\mathbf{q}}_{\mathrm{m}}, \dot{\mathbf{x}}_{\mathrm{m}}, \ddot{\mathbf{x}}_{\mathrm{m}}\right) \boldsymbol{\theta}_{\mathrm{md}} \\
& \mathbf{M}_{\mathrm{s}}\left(\mathbf{q}_{\mathrm{s}}\right) \ddot{\mathbf{x}}_{\mathrm{s}}+\mathbf{C}_{\mathrm{s}}\left(\mathbf{q}_{\mathrm{s}}, \dot{\mathbf{q}}_{\mathrm{s}}\right) \dot{\mathbf{x}}_{\mathrm{s}}+\mathbf{G}_{\mathrm{s}}\left(\mathbf{q}_{\mathrm{s}}\right) \\
& \quad=\mathbf{Y}_{\mathrm{sd}}\left(\mathbf{q}_{\mathrm{s}}, \dot{\mathbf{q}}_{\mathrm{s}}, \dot{\mathbf{x}}_{\mathrm{s}}, \ddot{\mathbf{x}}_{\mathrm{s}}\right) \boldsymbol{\theta}_{\mathrm{sd}}
\end{aligned}
$$

where $\mathbf{Y}_{\mathrm{md}} \in \mathrm{R}^{\mathrm{n} \times \mathrm{p}_{1}}$ and $\mathbf{Y}_{\mathrm{sd}} \in \mathrm{R}^{\mathrm{n} \times \mathrm{p}_{2}}$ are two dynamic regressor matrices.

\section{B. Design of Adaptive Bilateral Controllers}

When the dynamics of the master and the slave experience parametric uncertainties, the left sides of (1) and (2) become

$\widehat{\mathbf{M}}_{\mathrm{m}}\left(\mathbf{q}_{\mathrm{m}}\right) \ddot{\mathbf{x}}_{\mathrm{m}}+\widehat{\mathbf{C}}_{\mathrm{m}}\left(\mathbf{q}_{\mathrm{m}}, \dot{\mathbf{q}}_{\mathrm{m}}\right) \dot{\mathbf{x}}_{\mathrm{m}}+\widehat{\mathbf{G}}_{\mathrm{m}}\left(\mathbf{q}_{\mathrm{m}}\right)=\mathbf{Y}_{\mathrm{md}} \widehat{\boldsymbol{\theta}}_{\mathrm{md}}$

$\widehat{\mathbf{M}}_{\mathrm{s}}\left(\mathbf{q}_{\mathrm{s}}\right) \ddot{\mathbf{x}}_{\mathrm{s}}+\widehat{\mathbf{C}}_{\mathrm{s}}\left(\mathbf{q}_{\mathrm{s}}, \dot{\mathbf{q}}_{\mathrm{s}}\right) \dot{\mathbf{x}}_{\mathrm{s}}+\widehat{\mathbf{G}}_{\mathrm{s}}\left(\mathbf{q}_{\mathrm{s}}\right)=\mathbf{Y}_{\mathrm{sd}} \widehat{\boldsymbol{\theta}}_{\mathrm{sd}}$

where $\hat{\theta}_{\mathrm{md}}$ and $\hat{\theta}_{\mathrm{sd}}$ are the dynamic estimations of $\theta_{\mathrm{md}}$ and $\theta_{\text {sd }}$, respectively.

We are now in a position to propose our adaptive bilateral control algorithm for the above teleoperation system.

- Control laws for the master and the slave:

$$
\begin{aligned}
\mathbf{f}_{\mathrm{m}}= & \widehat{\mathbf{M}}_{\mathrm{m}}\left(\mathbf{q}_{\mathrm{m}}\right)\left(\ddot{\mathbf{x}}_{\mathrm{s}}-\mathrm{K}_{\mathrm{mv}} \Delta \dot{\mathbf{x}}_{\mathrm{m}}-\mathrm{K}_{\mathrm{mp}} \Delta \mathbf{x}_{\mathrm{m}}\right) \\
& +\widehat{\mathbf{C}}_{\mathrm{m}}\left(\mathbf{q}_{\mathrm{m}}, \dot{\mathbf{q}}_{\mathrm{m}}\right) \dot{\mathbf{x}}_{\mathrm{m}}+\widehat{\mathbf{G}}_{\mathrm{m}}\left(\mathbf{q}_{\mathrm{m}}\right) \\
& +\widehat{\mathbf{M}}_{\mathrm{m}}\left(\mathbf{q}_{\mathrm{m}}\right) \mathrm{C}_{2}\left(\mathbf{f}_{\mathrm{h}}-\mathbf{f}_{\mathrm{e}}\right)-\mathbf{f}_{\mathrm{h}} \\
\mathbf{f}_{\mathrm{s}}= & \widehat{\mathbf{M}}_{\mathrm{s}}\left(\mathbf{q}_{\mathrm{s}}\right)\left(\ddot{\mathbf{x}}_{\mathrm{m}}-\mathrm{K}_{\mathrm{sv}} \Delta \dot{\mathbf{x}}_{\mathrm{s}}-\mathrm{K}_{\mathrm{sp}} \Delta \mathbf{x}_{\mathrm{s}}\right) \\
& +\widehat{\mathbf{C}}_{\mathrm{s}}\left(\mathbf{q}_{\mathrm{s}}, \dot{\mathbf{q}}_{\mathrm{s}}\right) \dot{\mathbf{x}}_{\mathrm{s}}+\widehat{\mathbf{G}}_{\mathrm{s}}\left(\mathbf{q}_{\mathrm{s}}\right) \\
& +\widehat{\mathbf{M}}_{\mathrm{s}}\left(\mathbf{q}_{\mathrm{s}}\right) \mathrm{C}_{3}\left(\mathbf{f}_{\mathrm{h}}-\mathbf{f}_{\mathrm{e}}\right)+\mathbf{f}_{\mathrm{e}}
\end{aligned}
$$

where $\Delta \mathbf{x}_{\mathrm{m}}=\mathbf{x}_{\mathrm{m}}-\mathbf{x}_{\mathrm{s}}, \Delta \mathbf{x}_{\mathrm{s}}=\mathbf{x}_{\mathrm{s}}-\mathbf{x}_{\mathrm{m}}$, and $\mathrm{K}_{\mathrm{mv}}, \mathrm{K}_{\mathrm{sv}}, \mathrm{K}_{\mathrm{mp}}$, $\mathrm{K}_{\mathrm{sp}}, \mathrm{C}_{2}$ and $\mathrm{C}_{3}$ can all be chosen to be positive constants.

- Dynamic update laws:

$\dot{\widehat{\boldsymbol{\theta}}}_{\mathrm{md}}=-\mathbf{L}_{\mathrm{md}} \boldsymbol{\Phi}_{\mathrm{m}}^{\mathrm{T}}\left(\Delta \dot{\mathbf{x}}_{\mathrm{m}}+\alpha \Delta \mathbf{x}_{\mathrm{m}}\right)$

$\dot{\hat{\boldsymbol{\theta}}}_{\mathrm{sd}}=-\mathbf{L}_{\mathrm{sd}} \boldsymbol{\Phi}_{\mathrm{s}}^{\mathrm{T}}\left(\Delta \dot{\mathbf{x}}_{\mathrm{s}}+\alpha \Delta \mathbf{x}_{\mathrm{s}}\right)$

where $\alpha$ is a positive constant and is chosen such that $\alpha<\mathrm{K}_{\mathrm{v}}$ ( $\mathrm{K}_{\mathrm{v}}$ will be defined later). Also, $\mathbf{L}_{\mathrm{md}}$ and $\mathbf{L}_{\mathrm{sd}}$ are positive-definite matrices and

$\boldsymbol{\Phi}_{\mathrm{m}}=\left(1 /\left(1+\mathrm{C}_{2} / \mathrm{C}_{3}\right)\right) \widehat{\mathbf{M}}_{\mathrm{m}}^{-1}\left(\mathbf{q}_{\mathrm{m}}\right) \mathbf{Y}_{\mathrm{md}}$

$\boldsymbol{\Phi}_{\mathrm{s}}=\left(1 /\left(1+\mathrm{C}_{2} / \mathrm{C}_{3}\right)\right)\left(\mathrm{C}_{2} / \mathrm{C}_{3}\right) \widehat{\mathbf{M}}_{\mathrm{s}}^{-1}\left(\mathbf{q}_{\mathrm{s}}\right) \mathbf{Y}_{\mathrm{sd}}$

and $\Delta \boldsymbol{\theta}_{\mathrm{md}}=\widehat{\boldsymbol{\theta}}_{\mathrm{md}}-\boldsymbol{\theta}_{\mathrm{md}}$ and $\Delta \boldsymbol{\theta}_{\mathrm{sd}}=\widehat{\boldsymbol{\theta}}_{\mathrm{sd}}-\boldsymbol{\theta}_{\mathrm{sd}}$.

Each of the control laws (5)-(6) includes five parts. The first part, second part and the third part perform adaptive inverse dynamics position control (see Fig. 2). The fourth part involves force feedback. The fifth part compensates for the force due to the operator (for the master) and due to the environment (for the slave).

Substituting (5)-(6) into (1)-(2), the closed-loop equations of the master and the slave are obtained as

$$
\begin{aligned}
& \mathbf{M}_{\mathrm{m}}\left(\mathbf{q}_{\mathrm{m}}\right) \ddot{\mathbf{x}}_{\mathrm{m}}+\mathbf{C}_{\mathrm{m}}\left(\mathbf{q}_{\mathrm{m}}, \dot{\mathbf{q}}_{\mathrm{m}}\right) \dot{\mathbf{x}}_{\mathrm{m}}+\mathbf{G}_{\mathrm{m}}\left(\mathbf{q}_{\mathrm{m}}\right) \\
& \quad=\widehat{\mathbf{M}}_{\mathrm{m}}\left(\mathbf{q}_{\mathrm{m}}\right)\left(\ddot{\mathbf{x}}_{\mathrm{s}}-\mathrm{K}_{\mathrm{mv}} \Delta \dot{\mathbf{x}}_{\mathrm{m}}-\mathrm{K}_{\mathrm{mp}} \Delta \mathbf{x}_{\mathrm{m}}\right) \\
& \quad+\widehat{\mathbf{C}}_{\mathrm{m}}\left(\mathbf{q}_{\mathrm{m}}, \dot{\mathbf{q}}_{\mathrm{m}}\right) \dot{\mathbf{x}}_{\mathrm{m}}+\widehat{\mathbf{G}}_{\mathrm{m}}\left(\mathbf{q}_{\mathrm{m}}\right)+\widehat{\mathbf{M}}_{\mathrm{m}}\left(\mathrm{q}_{\mathrm{m}}\right) \mathrm{C}_{2}\left(\mathbf{f}_{\mathrm{h}}-\mathbf{f}_{\mathrm{e}}\right) \\
& \mathbf{M}_{\mathrm{s}}\left(\mathbf{q}_{\mathrm{s}}\right) \ddot{\mathbf{x}}_{\mathrm{s}}+\mathbf{C}_{\mathrm{s}}\left(\mathbf{q}_{\mathrm{s}}, \dot{\mathbf{q}}_{\mathrm{s}}\right) \dot{\mathbf{x}}_{\mathrm{s}}+\mathbf{G}_{\mathrm{s}}\left(\mathbf{q}_{\mathrm{s}}\right) \\
& \quad=\widehat{\mathbf{M}}_{\mathrm{s}}\left(\mathbf{q}_{\mathrm{s}}\right)\left(\ddot{\mathbf{x}}_{\mathrm{m}}-\mathrm{K}_{\mathrm{sv}} \Delta \dot{\mathbf{x}}_{\mathrm{s}}-\mathrm{K}_{\mathrm{sp}} \Delta \mathbf{x}_{\mathrm{s}}\right) \\
& \quad+\widehat{\mathbf{C}}_{\mathrm{s}}\left(\mathbf{q}_{\mathrm{s}}, \dot{\mathbf{q}}_{\mathrm{s}}\right) \dot{\mathbf{x}}_{\mathrm{s}}+\widehat{\mathbf{G}}_{\mathrm{s}}\left(\mathbf{q}_{\mathrm{s}}\right)+\widehat{\mathbf{M}}_{\mathrm{s}}\left(\mathrm{q}_{\mathrm{s}}\right) \mathrm{C}_{3}\left(\mathbf{f}_{\mathrm{h}}-\mathbf{f}_{\mathrm{e}}\right)
\end{aligned}
$$

Adding $\widehat{\mathbf{M}}_{\mathrm{m}}\left(\mathbf{q}_{\mathrm{m}}\right) \ddot{\mathbf{x}}_{\mathrm{m}}$ and then subtracting it in the right side of (9) helps to rewrite it as

$$
\begin{array}{r}
\widehat{\mathbf{M}}_{\mathrm{m}}\left(\mathbf{q}_{\mathrm{m}}\right)\left(\Delta \ddot{\mathbf{x}}_{\mathrm{m}}+\mathrm{K}_{\mathrm{mv}} \Delta \dot{\mathbf{x}}_{\mathrm{m}}+\mathrm{K}_{\mathrm{mp}} \Delta \mathbf{x}_{\mathrm{m}}\right) \\
=\mathbf{Y}_{\mathrm{md}} \Delta \boldsymbol{\theta}_{\mathrm{md}}+\widehat{\mathbf{M}}_{\mathrm{m}}\left(\mathbf{q}_{\mathrm{m}}\right) \mathrm{C}_{2}\left(\mathbf{f}_{\mathrm{h}}-\mathbf{f}_{\mathrm{e}}\right)
\end{array}
$$

Similarly, (10) can be rewritten as

$$
\begin{aligned}
\widehat{\mathbf{M}}_{\mathrm{s}}\left(\mathbf{q}_{\mathrm{s}}\right)\left(\Delta \ddot{\mathbf{x}}_{\mathrm{s}}+\mathrm{K}_{\mathrm{sv}} \Delta \dot{\mathbf{x}}_{\mathrm{s}}+\mathrm{K}_{\mathrm{sp}} \Delta \mathbf{x}_{\mathrm{s}}\right) \\
\quad=\mathbf{Y}_{\mathrm{sd}} \Delta \boldsymbol{\theta}_{\mathrm{sd}}+\widehat{\mathbf{M}}_{\mathrm{s}}\left(\mathbf{q}_{\mathrm{s}}\right) \mathrm{C}_{3}\left(\mathbf{f}_{\mathrm{h}}-\mathbf{f}_{\mathrm{e}}\right)
\end{aligned}
$$

Multiplying both sides of (11) and (12) by $\widehat{\mathbf{M}}_{\mathrm{m}}^{-1}\left(\mathbf{q}_{\mathrm{m}}\right)$ and $\widehat{\mathbf{M}}_{\mathrm{s}}^{-1}\left(\mathbf{q}_{\mathrm{s}}\right)$, respectively, gives

$$
\begin{aligned}
& \Delta \ddot{\mathbf{x}}_{\mathrm{m}}+\mathrm{K}_{\mathrm{mv}} \Delta \dot{\mathbf{x}}_{\mathrm{m}}+\mathrm{K}_{\mathrm{mp}} \Delta \mathbf{x}_{\mathrm{m}}-\widehat{\mathbf{M}}_{\mathrm{m}}^{-1}\left(\mathbf{q}_{\mathrm{m}}\right) \mathbf{Y}_{\mathrm{md}} \Delta \boldsymbol{\theta}_{\mathrm{md}} \\
& \quad=\mathrm{C}_{2}\left(\mathbf{f}_{\mathrm{h}}-\mathbf{f}_{\mathrm{e}}\right) \\
& \begin{aligned}
\Delta \ddot{\mathbf{x}}_{\mathrm{s}} & +\mathrm{K}_{\mathrm{sv}} \Delta \dot{\mathbf{x}}_{\mathrm{s}}+\mathrm{K}_{\mathrm{sp}} \Delta \mathbf{x}_{\mathrm{s}}-\widehat{\mathbf{M}}_{\mathrm{s}}^{-1}\left(\mathbf{q}_{\mathrm{s}}\right) \mathbf{Y}_{\mathrm{sd}} \Delta \boldsymbol{\theta}_{\mathrm{sd}} \\
= & \mathrm{C}_{3}\left(\mathbf{f}_{\mathrm{h}}-\mathbf{f}_{\mathrm{e}}\right)
\end{aligned}
\end{aligned}
$$

Now, multiplying both sides of (14) by $\mathrm{C}_{2} / \mathrm{C}_{3}$, using $\Delta \mathbf{x}_{\mathrm{m}}=-\Delta \mathbf{x}_{\mathrm{s}}$, and subtracting (13) from the result, we arrive at the following closed-loop dynamics for the master and the slave:

$\left(1+\mathrm{C}_{2} / \mathrm{C}_{3}\right) \Delta \ddot{\mathbf{x}}_{\mathrm{m}}+\left(\mathrm{K}_{\mathrm{mv}}+\left(\mathrm{C}_{2} / \mathrm{C}_{3}\right) \mathbf{K}_{\mathrm{sv}}\right) \Delta \dot{\mathbf{x}}_{\mathrm{m}}$

$+\left(\mathrm{K}_{\mathrm{mp}}+\left(\mathrm{C}_{2} / \mathrm{C}_{3}\right) \mathrm{K}_{\mathrm{sp}}\right) \Delta \mathbf{x}_{\mathrm{m}}-\widehat{\mathbf{M}}_{\mathrm{m}}^{-1}\left(\mathbf{q}_{\mathrm{m}}\right) \mathbf{Y}_{\mathrm{md}} \Delta \boldsymbol{\theta}_{\mathrm{md}}$

$+\left(\mathrm{C}_{2} / \mathrm{C}_{3}\right) \widehat{\mathbf{M}}_{\mathrm{s}}^{-1}\left(\mathbf{q}_{\mathrm{s}}\right) \mathbf{Y}_{\mathrm{sd}} \Delta \boldsymbol{\theta}_{\mathrm{sd}}=0$

Multiplying both sides of $(15)$ by $1 /\left(1+C_{2} / C_{3}\right)$, this unified closed-loop dynamics can be rewritten as

$\Delta \ddot{\mathbf{x}}_{\mathrm{m}}+\mathrm{K}_{\mathrm{v}} \Delta \dot{\mathbf{x}}_{\mathrm{m}}+\mathrm{K}_{\mathrm{p}} \Delta \mathbf{x}_{\mathrm{m}}=\boldsymbol{\Phi}_{\mathrm{m}} \Delta \boldsymbol{\theta}_{\mathrm{md}}-\boldsymbol{\Phi}_{\mathrm{s}} \Delta \boldsymbol{\theta}_{\mathrm{sd}}$

where

$$
\begin{aligned}
& \mathrm{K}_{\mathrm{v}}=1 /\left(1+\mathrm{C}_{2} / \mathrm{C}_{3}\right)\left(\mathrm{K}_{\mathrm{mv}}+\left(\mathrm{C}_{2} / \mathrm{C}_{3}\right) \mathrm{K}_{\mathrm{sv}}\right) \\
& \mathrm{K}_{\mathrm{p}}=1 /\left(1+\mathrm{C}_{2} / \mathrm{C}_{3}\right)\left(\mathrm{K}_{\mathrm{mp}}+\left(\mathrm{C}_{2} / \mathrm{C}_{3}\right) \mathrm{K}_{\mathrm{sp}}\right)
\end{aligned}
$$

Since $\mathrm{K}_{\mathrm{mv}}, \mathrm{K}_{\mathrm{sv}}, \mathrm{K}_{\mathrm{mp}}, \mathrm{K}_{\mathrm{sp}}, \mathrm{C}_{2}$ and $\mathrm{C}_{3}$ are all positive constants, $K_{v}$ and $K_{p}$ are also positive constants. It can be seen that the left side of (16) is a linear error expression. 
Remark 1: A potential issue with control laws (5)-(6) is the assumption that the accelerations $\ddot{\mathrm{x}}_{\mathrm{m}}$ and $\ddot{\mathrm{x}}_{\mathrm{s}}$ are measured. The same assumption is regularly made for achieving full transparency in the original 4-channel teleoperation architecture. Indeed, the controllers $C_{1}$ and $C_{4}$ in the original 4-channel design require acceleration measurements to be able to guarantee asymptotic position tracking $\left(\ddot{e}+k_{v} \dot{e}+\right.$ $k_{p} e=0$, where $e$ is the position error) given the inertia contributions of the master and slave dynamics [2]. It must be noted, however, that near-transparency can be obtained at low frequencies by ignoring these acceleration terms. Indeed, since voluntary motions of the human hand are themselves band-limited $^{1}$, in the absence of acceleration measurements, position and force tracking will be good sort of feeling high-frequency phenomena such as the sharp edges or texture of an object. On the other hand, if perfect transparency over a large bandwidth is required, using accelerometers may be justifiable. Alternatively, it is possible to use position measurements with differentiators that are robust to measurement noise. For instance, Levant [17] designed a robust exact differentiator, and Suzuki et al [18] proposed an adaptive version of Levant's differentiator. Sidhom et al [19] dealt with the use of Suzuki's differentiator in an identification context.

Remark 2: The estimated inertia matrices $\widehat{M}_{m}\left(q_{m}\right)$ and $\widehat{M}_{s}\left(q_{s}\right)$ for the master and the slave are assumed to be invertible. To remove this assumption, in the controllers (5)-(6), $\widehat{M}_{m}\left(q_{m}\right)$ and $\widehat{M}_{s}\left(q_{s}\right)$ can be replaced by their priori estimates $\widehat{\mathrm{M}}_{\mathrm{mp}}$ and $\widehat{\mathrm{M}}_{\mathrm{sp}}$, respectively, where $\widehat{\mathrm{M}}_{\mathrm{mp}}=\widehat{\mathrm{M}}_{\mathrm{mp}}^{\mathrm{T}} \geq$ 0 and $\widehat{M}_{\mathrm{sp}}=\widehat{\mathrm{M}}_{\mathrm{sp}}^{\mathrm{T}} \geq 0$. Since $\widehat{\mathrm{M}}_{\mathrm{mp}}$ and $\widehat{\mathrm{M}}_{\mathrm{sp}}$ are not updated online, the invertibility of $\widehat{\mathrm{M}}_{\mathrm{mp}}$ and $\widehat{\mathrm{M}}_{\mathrm{sp}}$ is not a concern. More details about this can be found in [11], [13].

\section{TRANSPARENCY OF Closed-LOOP SYSTEM}

In an ideally transparent teleoperation system, through appropriate control signals $\mathbf{f}_{\mathrm{m}}$ and $\mathbf{f}_{\mathrm{s}}$, the master and the slave positions and forces will match regardless of the operator and environment dynamics, i.e., $\mathbf{x}_{\mathrm{m}}=\mathbf{x}_{\mathrm{s}}$ and $\mathbf{f}_{\mathrm{h}}=\mathbf{f}_{\mathrm{e}}$ [20]. Since, we have obtained the unified closed-loop dynamics (16), we can use a unified Lyapunov function to show the transparency of the overall system as in this section.

Theorem 1: Consider the nonlinear teleoperation system (1)-(2), which has dynamic uncertainty and is controlled by the adaptive controller (5)-(6) using the dynamic update laws (7)-(8). Then, the signals $\Delta \mathbf{x}_{\mathrm{m}}, \dot{\mathbf{x}}_{\mathrm{m}}, \Delta \boldsymbol{\theta}_{\mathrm{md}}$ and $\Delta \boldsymbol{\theta}_{\mathrm{sd}}$ in the closed-loop system are bounded. Moreover, the position tracking error $\Delta \mathbf{x}_{\mathrm{m}}=\mathbf{x}_{\mathrm{m}}-\mathbf{x}_{\mathrm{s}}$ converges to zero as $\mathrm{t} \rightarrow \infty$, and the force tracking error $\Delta \mathbf{f}=\mathbf{f}_{\mathrm{h}}-\mathbf{f}_{\mathrm{e}}$ is bounded.

\footnotetext{
${ }^{1}$ The maximum bandwidth with which the human finger can apply motion or force commands is $5-10 \mathrm{~Hz}[15]$ and the maximum bandwidth with which the human finger reacts to tactile stimuli is $8-10 \mathrm{~Hz}[16]$.
}

Proof: Consider a Lyapunov function candidate as

$$
\begin{aligned}
\mathrm{V}= & \frac{1}{2}\left(\Delta \dot{\mathbf{x}}_{\mathrm{m}}+\alpha \Delta \mathbf{x}_{\mathrm{m}}\right)^{\mathrm{T}}\left(\Delta \dot{\mathbf{x}}_{\mathrm{m}}+\alpha \Delta \mathbf{x}_{\mathrm{m}}\right) \\
& +\frac{1}{2} \Delta \mathbf{x}_{\mathrm{m}}^{\mathrm{T}}\left(\mathrm{K}_{\mathrm{p}}+\alpha \mathrm{K}_{\mathrm{v}}-\alpha^{2}\right) \Delta \mathbf{x}_{\mathrm{m}}+\frac{1}{2} \Delta \boldsymbol{\theta}_{\mathrm{md}}^{\mathrm{T}} \mathbf{L}_{\mathrm{md}}^{-1} \Delta \boldsymbol{\theta}_{\mathrm{md}} \\
& +\frac{1}{2} \Delta \boldsymbol{\theta}_{\mathrm{sd}}^{\mathrm{T}} \mathbf{L}_{\mathrm{sd}}^{-1} \Delta \boldsymbol{\theta}_{\mathrm{sd}}
\end{aligned}
$$

where $\alpha$ is a positive constant and is chosen such that $\alpha<\mathrm{K}_{\mathrm{V}}$. The derivative of $\mathrm{V}$ along the trajectory of the unified closed-loop system (16) is

$$
\begin{aligned}
\dot{\mathrm{V}}= & \left(\Delta \dot{\mathbf{x}}_{\mathrm{m}}+\alpha \Delta \mathbf{x}_{\mathrm{m}}\right)^{\mathrm{T}}\left(-\mathrm{K}_{\mathrm{v}} \Delta \dot{\mathbf{x}}_{\mathrm{m}}-\mathrm{K}_{\mathrm{p}} \Delta \mathbf{x}_{\mathrm{m}}+\boldsymbol{\Phi}_{\mathrm{m}} \Delta \boldsymbol{\theta}_{\mathrm{md}}\right. \\
& \left.-\boldsymbol{\Phi}_{\mathrm{s}} \Delta \boldsymbol{\theta}_{\mathrm{sd}}+\alpha \Delta \dot{\mathbf{x}}_{\mathrm{m}}\right)+\Delta \dot{\mathbf{x}}_{\mathrm{m}}^{\mathrm{T}}\left(\mathrm{K}_{\mathrm{p}}+\alpha \mathrm{K}_{\mathrm{v}}-\alpha^{2}\right) \Delta \mathbf{x}_{\mathrm{m}} \\
& +\Delta \boldsymbol{\theta}_{\mathrm{md}}^{\mathrm{T}} \mathbf{L}_{\mathrm{md}}^{-1} \dot{\widehat{\boldsymbol{\theta}}}_{\mathrm{md}}+\Delta \boldsymbol{\theta}_{\mathrm{sd}}^{\mathrm{T}} \mathbf{L}_{\mathrm{sd}}^{-1} \dot{\widehat{\boldsymbol{\theta}}}_{\mathrm{sd}}
\end{aligned}
$$

Since

$$
\begin{array}{r}
-\mathrm{K}_{\mathrm{v}} \Delta \dot{\mathbf{x}}_{\mathrm{m}}-\mathrm{K}_{\mathrm{p}} \Delta \mathbf{x}_{\mathrm{m}}+\boldsymbol{\Phi}_{\mathrm{m}} \Delta \boldsymbol{\theta}_{\mathrm{md}}-\boldsymbol{\Phi}_{\mathrm{s}} \Delta \boldsymbol{\theta}_{\mathrm{sd}}+\alpha \Delta \dot{\mathbf{x}}_{\mathrm{m}} \\
=-\left(\mathrm{K}_{\mathrm{v}}-\alpha\right) \Delta \dot{\mathbf{x}}_{\mathrm{m}}-\mathrm{K}_{\mathrm{p}} \Delta \mathbf{x}_{\mathrm{m}}+\boldsymbol{\Phi}_{\mathrm{m}} \Delta \boldsymbol{\theta}_{\mathrm{md}}-\boldsymbol{\Phi}_{\mathrm{s}} \Delta \boldsymbol{\theta}_{\mathrm{sd}}
\end{array}
$$

we get

$$
\begin{aligned}
\dot{\mathrm{V}}= & \left(\Delta \dot{\mathbf{x}}_{\mathrm{m}}+\alpha \Delta \mathbf{x}_{\mathrm{m}}\right)^{\mathrm{T}} \\
& \left(-\left(\mathrm{K}_{\mathrm{v}}-\alpha\right) \Delta \dot{\mathbf{x}}_{\mathrm{m}}-\mathrm{K}_{\mathrm{p}} \Delta \mathbf{x}_{\mathrm{m}}+\boldsymbol{\Phi}_{\mathrm{m}} \Delta \boldsymbol{\theta}_{\mathrm{md}}-\boldsymbol{\Phi}_{\mathrm{s}} \Delta \boldsymbol{\theta}_{\mathrm{sd}}\right) \\
& +\Delta \dot{\mathbf{x}}_{\mathrm{m}}^{\mathrm{T}}\left(\mathrm{K}_{\mathrm{p}}+\alpha \mathrm{K}_{\mathrm{v}}-\alpha^{2}\right) \Delta \mathbf{x}_{\mathrm{m}}+\Delta \boldsymbol{\theta}_{\mathrm{md}}^{\mathrm{T}} \mathbf{L}_{\mathrm{md}}^{-1} \dot{\widehat{\boldsymbol{\theta}}}_{\mathrm{md}} \\
& +\Delta \boldsymbol{\theta}_{\mathrm{sd}}^{\mathrm{T}} \mathbf{L}_{\mathrm{sd}}^{-1} \dot{\boldsymbol{\theta}}_{\mathrm{sd}}
\end{aligned}
$$

Also, since

$$
\begin{gathered}
\left(\Delta \dot{\mathbf{x}}_{\mathrm{m}}+\alpha \Delta \mathbf{x}_{\mathrm{m}}\right)^{\mathrm{T}}\left(-\left(\mathrm{K}_{\mathrm{v}}-\alpha\right) \Delta \dot{\mathbf{x}}_{\mathrm{m}}-\mathrm{K}_{\mathrm{p}} \Delta \mathbf{x}_{\mathrm{m}}\right) \\
=-\Delta \dot{\mathbf{x}}_{\mathrm{m}}^{\mathrm{T}}\left(\mathrm{K}_{\mathrm{v}}-\alpha\right) \Delta \dot{\mathbf{x}}_{\mathrm{m}}-\alpha \Delta \mathbf{x}_{\mathrm{m}}^{\mathrm{T}} \mathrm{K}_{\mathrm{p}} \Delta \mathbf{x}_{\mathrm{m}} \\
-\Delta \dot{\mathbf{x}}_{\mathrm{m}}^{\mathrm{T}}\left(\mathrm{K}_{\mathrm{p}}+\alpha \mathrm{K}_{\mathrm{v}}-\alpha^{2}\right) \Delta \mathbf{x}_{\mathrm{m}},
\end{gathered}
$$

we have

$$
\begin{aligned}
\dot{\mathrm{V}}= & -\Delta \dot{\mathbf{x}}_{\mathrm{m}}^{\mathrm{T}}\left(\mathrm{K}_{\mathrm{v}}-\alpha\right) \Delta \dot{\mathbf{x}}_{\mathrm{m}}-\alpha \Delta \mathbf{x}_{\mathrm{m}}^{\mathrm{T}} \mathrm{K}_{\mathrm{p}} \Delta \mathbf{x}_{\mathrm{m}} \\
& -\Delta \dot{\mathbf{x}}_{\mathrm{m}}^{\mathrm{T}}\left(\mathrm{K}_{\mathrm{p}}+\alpha \mathrm{K}_{\mathrm{v}}-\alpha^{2}\right) \Delta \mathbf{x}_{\mathrm{m}} \\
& +\Delta \dot{\mathbf{x}}_{\mathrm{m}}^{\mathrm{T}}\left(\mathrm{K}_{\mathrm{p}}+\alpha \mathrm{K}_{\mathrm{v}}-\alpha^{2}\right) \Delta \mathbf{x}_{\mathrm{m}} \\
& +\left(\Delta \dot{\mathbf{x}}_{\mathrm{m}}+\alpha \Delta \mathbf{x}_{\mathrm{m}}\right)^{\mathrm{T}} \boldsymbol{\Phi}_{\mathrm{m}} \Delta \boldsymbol{\theta}_{\mathrm{md}} \\
& -\left(\Delta \dot{\mathbf{x}}_{\mathrm{m}}+\alpha \Delta \mathbf{x}_{\mathrm{m}}\right)^{\mathrm{T}} \boldsymbol{\Phi}_{\mathrm{s}} \Delta \boldsymbol{\theta}_{\mathrm{sd}} \\
& +\Delta \boldsymbol{\theta}_{\mathrm{md}}^{\mathrm{T}} \mathbf{L}_{\mathrm{md}}^{-1} \dot{\hat{\boldsymbol{\theta}}}_{\mathrm{md}}+\Delta \boldsymbol{\theta}_{\mathrm{sd}}^{\mathrm{T}} \mathbf{L}_{\mathrm{sd}}^{-1} \dot{\boldsymbol{\theta}}_{\mathrm{sd}} \\
= & -\Delta \dot{\mathbf{x}}_{\mathrm{m}}^{\mathrm{T}}\left(\mathrm{K}_{\mathrm{v}}-\alpha\right) \Delta \dot{\mathbf{x}}_{\mathrm{m}}-\alpha \Delta \mathbf{x}_{\mathrm{m}}^{\mathrm{T}} \mathrm{K}_{\mathrm{p}} \Delta \mathbf{x}_{\mathrm{m}} \\
& +\left(\Delta \dot{\mathbf{x}}_{\mathrm{m}}+\alpha \Delta \mathbf{x}_{\mathrm{m}}\right)^{\mathrm{T}} \boldsymbol{\Phi}_{\mathrm{m}} \Delta \boldsymbol{\theta}_{\mathrm{md}} \\
& -\left(\Delta \dot{\mathbf{x}}_{\mathrm{m}}+\alpha \Delta \mathbf{x}_{\mathrm{m}}\right)^{\mathrm{T}} \boldsymbol{\Phi}_{\mathrm{s}} \Delta \boldsymbol{\theta}_{\mathrm{sd}} \\
& +\Delta \boldsymbol{\theta}_{\mathrm{md}}^{\mathrm{T}} \mathbf{L}_{\mathrm{md}}^{-1} \dot{\boldsymbol{\theta}}_{\mathrm{md}}+\Delta \boldsymbol{\theta}_{\mathrm{sd}}^{\mathrm{T}} \mathbf{L}_{\mathrm{sd}}^{-1} \dot{\boldsymbol{\theta}}_{\mathrm{sd}}
\end{aligned}
$$

Substituting the adaptation laws (7)-(8) into (18), it becomes

$\dot{\mathrm{V}}=-\Delta \dot{\mathbf{x}}_{\mathrm{m}}^{\mathrm{T}}\left(\mathrm{K}_{\mathrm{v}}-\alpha\right) \Delta \dot{\mathbf{x}}_{\mathrm{m}}-\alpha \Delta \mathbf{x}_{\mathrm{m}}^{\mathrm{T}} \mathrm{K}_{\mathrm{p}} \Delta \mathbf{x}_{\mathrm{m}} \leq 0$ 
Since $V$ is positive definite and $\dot{V}$ is negative semi-definite, $\mathrm{V}$ is bounded and the closed-loop system signals $\Delta \mathbf{x}_{\mathrm{m}}, \Delta \dot{\mathbf{x}}_{\mathrm{m}}$, $\Delta \boldsymbol{\theta}_{\mathrm{md}}$ and $\Delta \boldsymbol{\theta}_{\text {sd }}$ are bounded, i.e., they belong to $\mathrm{L}_{\infty}$.

For analyzing position tracking error convergence, integrating $\dot{V}$ gives us

$$
\begin{aligned}
\mathrm{V}(\mathrm{t}) & =\int_{0}^{\mathrm{t}} \dot{\mathrm{V}} \mathrm{dt} \\
& =-\left(\mathrm{K}_{\mathrm{v}}-\alpha\right) \int_{0}^{\mathrm{t}}\left\|\Delta \dot{\mathbf{x}}_{\mathrm{m}}\right\|^{2} \mathrm{dt}-\alpha \mathrm{K}_{\mathrm{p}} \int_{0}^{\mathrm{t}}\left\|\Delta \mathbf{x}_{\mathrm{m}}\right\|^{2} \mathrm{dt}
\end{aligned}
$$

Since $V(t)$ is bounded, as $\mathrm{t} \rightarrow \infty$, we get that $\int_{0}^{\infty}\left\|\Delta \mathbf{x}_{\mathrm{m}}\right\|^{2} \mathrm{dt}$ and $\int_{0}^{\infty}\left\|\Delta \dot{\mathbf{x}}_{\mathrm{m}}\right\|^{2} \mathrm{dt}$ are bounded, i.e., $\Delta \mathbf{x}_{\mathrm{m}}, \Delta \dot{\mathbf{x}}_{\mathrm{m}} \in \mathrm{L}_{2}$. Having $\Delta \mathbf{x}_{\mathrm{m}}, \Delta \dot{\mathbf{x}}_{\mathrm{m}} \in \mathrm{L}_{\infty}$ and $\Delta \mathbf{x}_{\mathrm{m}} \in \mathrm{L}_{2}$, and using the fact that a square integrable signal with a bounded derivative convergences to zero [9], we can obtain that $\lim _{\mathrm{t} \rightarrow \infty} \Delta \mathbf{x}_{\mathrm{m}}=$ $\lim _{t \rightarrow \infty}\left(\mathbf{x}_{\mathrm{m}}-\mathbf{x}_{\mathrm{s}}\right)=0$.

In terms of force tracking performance, since $\Delta \mathbf{x}_{\mathrm{m}}$, $\Delta \dot{\mathbf{x}}_{\mathrm{m}}, \Delta \boldsymbol{\theta}_{\mathrm{md}}$ and $\Delta \boldsymbol{\theta}_{\mathrm{sd}}$ are bounded, according to the unified closed-loop system (16), we can obtain that $\Delta \ddot{\mathrm{x}}_{\mathrm{m}}$ is bounded. Therefore, from (13), we find that the force tracking error $\Delta \mathbf{f}=\mathbf{f}_{\mathrm{h}}-\mathbf{f}_{\mathrm{e}}$ is bounded. This concludes the proof.

\section{Simulation Study}

In this section, simulations are conducted to illustrate the performance of the proposed controller. Consider the master and slave robots to be identical and 2-DOF planar manipulators [14], each consisting of two links and two rotary joints, as shown in Fig.3.

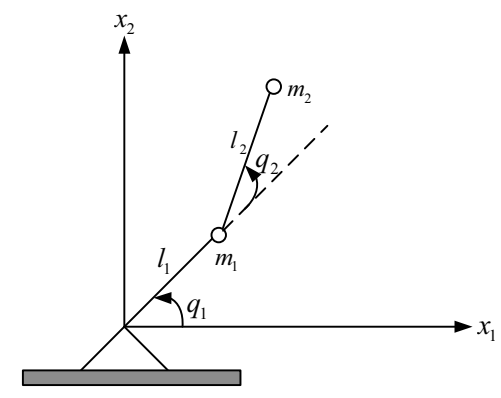

Fig. 3 2-DOF planar manipulator

The center of mass of each link is at the end of that link. The masses of the first and second links are $m_{1}$ and $m_{2}$, respectively, and the lengths of the two links are $l_{1}$ and $l_{2}$, respectively. The inertia matrix, and Coriolis and centrifugal term of the robot in the Cartesian space are as follows:

$$
\begin{aligned}
& \mathbf{M}(\mathbf{q})=\left[\begin{array}{cc}
\mathrm{m}_{2}+\frac{\mathrm{m}_{1}}{\left(\sin \left(\mathrm{q}_{2}\right)\right)^{2}} & 0 \\
0 & \mathrm{~m}_{2}
\end{array}\right], \\
& \mathbf{C}(\mathbf{q}, \dot{\mathbf{q}})=\left[\begin{array}{cc}
\mathrm{W} & -\mathrm{m}_{2} \dot{\mathrm{q}}_{2} \\
\mathrm{~m}_{2}\left(\dot{\mathrm{q}}_{1}+\mathrm{q}_{2}\right) & 0
\end{array}\right],
\end{aligned}
$$

where

$\mathrm{W}=\mathrm{l}_{1} \mathrm{~m}_{2}\left(\dot{\mathrm{q}}_{1} \cos \left(\mathrm{q}_{2}\right)-\dot{\mathrm{q}}_{1} \cos ^{3}\left(\mathrm{q}_{2}\right)\right)+\mathrm{l}_{1} \mathrm{~m}_{1} \dot{\mathrm{q}}_{2} \cos \left(\mathrm{q}_{2}\right)$

$$
+\mathrm{l}_{2} \mathrm{~m}_{2}\left(\dot{\mathrm{q}}_{1}-\dot{\mathrm{q}}_{1} \cos ^{2}\left(\mathrm{q}_{2}\right)+\dot{\mathrm{q}}_{2}-\dot{\mathrm{q}}_{2} \cos ^{2}\left(\mathrm{q}_{2}\right)\right) .
$$

The gravity term is assumed to be zero for simplicity. The unknown dynamic parameter vectors for the master and the slave are $\boldsymbol{\theta}_{\mathrm{md}}=\boldsymbol{\theta}_{\mathrm{sd}}=\left[\mathrm{m}_{2}, \mathrm{~m}_{1}, \mathrm{~m}_{2} \mathrm{l}_{2} / \mathrm{l}_{1}\right]^{\mathrm{T}}$, and the dynamic regressor matrices can be obtained according to Property 1 .

In the simulations, the exogenous force $\mathbf{f}_{\mathrm{h}}^{*}$ is taken as $[100 \sin 2 t, 0]^{\mathrm{T}}$. Other simulation parameters are

$\mathrm{m}_{1}=2.3 \mathrm{~kg}, \mathrm{~m}_{2}=4.6 \mathrm{~kg}, \mathrm{l}_{1}=\mathrm{l}_{2}=0.5 \mathrm{~m}$,

$\alpha=0.1, \mathrm{~K}_{\mathrm{mv}}=\mathrm{K}_{\mathrm{sv}}=1, \mathrm{~K}_{\mathrm{mp}}=\mathrm{K}_{\mathrm{sp}}=2$,

$\mathrm{C}_{2}=\mathrm{C}_{3}=0.05, \mathbf{L}_{\mathrm{md}}=\mathbf{L}_{\mathrm{sd}}=0.1 \mathrm{I}$, where $\mathbf{I}$ is the unit matrix. The initial positions of the master and slave robots are set as $\mathbf{x}_{\mathrm{m}}(0)=\mathbf{x}_{\mathrm{s}}(0)=[-0.05,0.3794]^{\mathrm{T}}$, and the initial estimations of the uncertain parameters are set as

$\widehat{\boldsymbol{\theta}}_{\mathrm{md}}(0)=\widehat{\boldsymbol{\theta}}_{\mathrm{sd}}(0)=[3.22,1.61,3.22]^{\mathrm{T}}$.

The simulation results in $\mathrm{x}_{1}$-direction are shown in Fig. 4(a)-(b). We can see that the slave tracks the position of the master well even though there are dynamic uncertainties in the teleoperation system. Besides, the force tracking error between the master and the slave is bounded and is very small. Since the exogenous force $\mathbf{f}_{\mathrm{h}}^{*}$ in the $\mathrm{x}_{2}$-direction is zero, perfect position and force tracking exists in that direction and, therefore, the results are not shown.

The performance of the proposed adaptive inverse dynamics control approach is also compared with the well-known conventional adaptive control approach in [5], which did not employ inverse dynamics (generating the results in Fig. 5(a)-(b)). Comparing Fig. 4 with Fig. 5, it can be found that the performance of the proposed adaptive inverse dynamics control approach is better.

Remark 3: It is worth noting that the estimated parameters $\widehat{\boldsymbol{\theta}}_{\mathrm{md}}, \widehat{\boldsymbol{\theta}}_{\mathrm{sd}}$ do not need to converge to their true values $\boldsymbol{\theta}_{\mathrm{md}}, \boldsymbol{\theta}_{\mathrm{sd}}$. A key point in adaptive control is that the tracking errors of the system may converge to zero regardless of whether the parameter errors converge to zero or not.

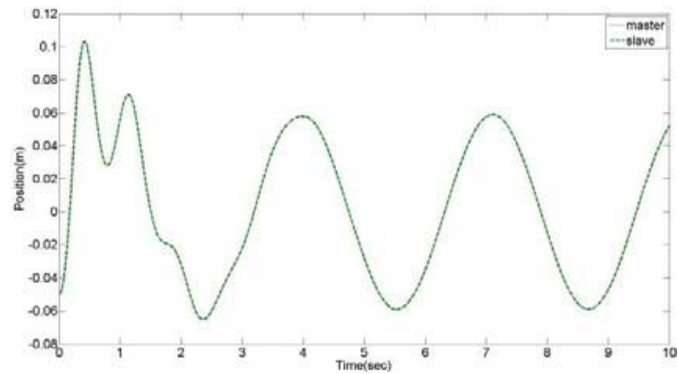

(a) Position tracking 


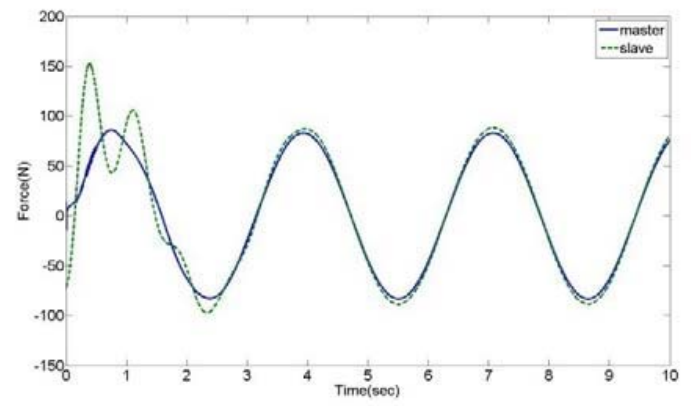

(b) Force tracking

Fig. 4. The proposed inverse dynamics-based adaptive control

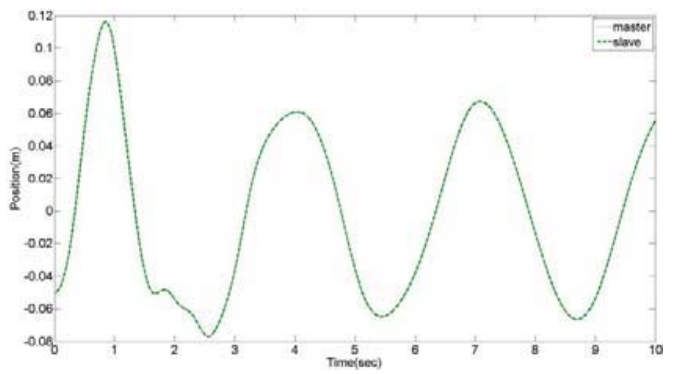

(a) Position tracking

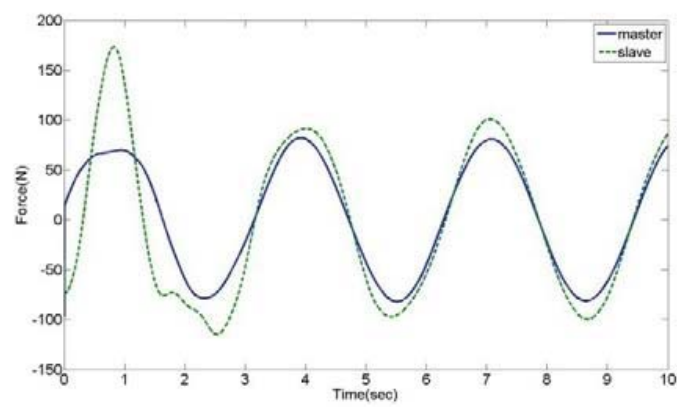

(c) Force tracking

Fig. 5. The adaptive control in [5]

\section{CONCLUSIONS}

In this paper, for multi-DOF teleoperation systems with uncertain nonlinear dynamics, adaptive inverse dynamics controllers are incorporated into Lawrence's 4-channel bilateral teleoperation framework. Compared with other adaptive control schemes for teleoperation systems, the proposed adaptive controller can yield linear error dynamics in the ideal case. Consequently, the transparency of the closed-loop system is more convenient to study.

With respect to force tracking, we have shown the force tracking error $\Delta \mathbf{f}=\mathbf{f}_{\mathrm{h}}-\mathbf{f}_{\mathrm{e}}$ is bounded. Improving the proposed control algorithm to ensure that the force tracking error converges to zero remains as future work.

\section{REFERENCES}

[1] P. Hokayem and M. Spong, "Bilateral teleoperation: an historical survey," Automatica, vol. 42, no. 12, pp. 2035-2057, 2006.
[2] D. A. Lawrence, "Stability and transparency in bilateral teleoperation," IEEE Transactions on Robotics and Automation, vol. 9, no. 5, pp. 624-637, 1993.

[3] Hyoung-Ki Lee and M. J. Chung, "Adaptive controller of a master-slave system for transparent teleoperation," Journal of Robotic systems, vol. 15, no. 8, pp. 465-475, 1998.

[4] M. Shi, G. Tao and H. Liu, "Adaptive control of teleoperation systems," Journal of X-Ray Science and Technology, vol. 10, no. 1-2, pp. 37-57, 2002.

[5] J. H. Ryu and D. S. Kwon, "A novel adaptive bilateral control scheme using similar closed-loop dynamic characteristics of master/slave manipulators," Journal of Robotic Systems, vol. 18, no. 9, pp. 533-543, 2001 .

[6] N. V. Q. Hung, T. Narikiyo and H. D. Tuan, "Nonlinear adaptive control of master-slave system in teleoperation," Control Engineering Practice, vol. 11, no. 1, pp. 1-10, 2003.

[7] Wen-Hong Zhu and S. E. Salcudean, "Stability guaranteed teleoperation: an adaptive motion/force control approach," IEEE transactions on automatic control, vol. 45, no. 11, pp. 1951-1969, 2000.

[8] P. Malysz and S. Sirouspour, "Nonlinear and filtered force/position mapping in bilateral teleoperation with application to enhanced stiffness discrimination," IEEE Transactions on Robotics, vol. 25, no. 5, pp. 1134-1149, 2009.

[9] N. Chopra, M. W. Spong and R. Lozano, "Synchronization of bilateral teleoperators with time delay," Automatica, vol. 44, no. 8, pp. 2142-2148, 2008.

[10] E. Nuño, R. Ortega and L. Basañez, "An adaptive controller for nonlinear teleoperators," Automatica, vol. 46, no. 1, pp. 155-159, 2010.

[11] M.W. Spong and R. Ortega, "On adaptive inverse dynamics control of rigid robots," IEEE Transactions on Automatic Control, vol. 35, no. 1, pp. 92-95, 1990.

[12] D. M. Dawson and F. L. Lewis, "Comments on "on adaptive inverse dynamics control of rigid robots,' "IEEE Transactions on Automatic Control, vol. 36, no. 10, pp. 1215-1216, 1991.

[13] H. Wang and Y. Xie, "Adaptive inverse dynamics control of robots with uncertain kinematics and dynamics," Automatica, vol. 45, no.9, pp. 2114-2119, 2009.

[14] J. Craig, Introduction to robotics: mechanics and control ( $3^{\text {rd }}$ Ed.), Pearson Prentice Hall, USA, 2005.

[15] K.B. Shimoga, "A survey of perceptual feedback issues in dexterous telemanipulation: Part I. Finger force feedback," Proceedings in IEEE Virtual Reality Annual International Symposium, pp. 263-270, 1993.

[16] K.B. Shimoga, "A survey of perceptual feedback issues in dexterous telemanipulation: Part II. Finger touch feedback," Proceedings in IEEE Virtual Reality Annual International Symposium, pp. 271-279, 1993.

[17] A. Levant, "Robust exact differentiation via sliding mode technique," Automatica, vol. 34, no. 3, pp. 379-384, 1998.

[18] S. Suzuki, K. Furuta, and S. Shiratori, "Adaptive impact shot control by pendulum-like juggling system," JSME International Journal, vol. 46, no. 3, pp. 973-981, 2003.

[19] L. Sidhom, M.T. Pham, F. Th'evenoux and M. Gautier, "Identification of a robot manipulator based on an adaptive higher order sliding modes differentiator," IEEE/ASME International Conference on Advanced Intelligent Mechatronics, Montréal, Canada, July, 2010.

[20] M. Tavakoli, A. Aziminejad, R.V. Patel and M. Moallem, "High-fidelity bilateral teleoperation systems and the effect of multimodal haptics," IEEE Transactions on Systems, Man, and Cybernetics - Part B, vol. 37, no.6, pp. 1512-1528, 2007. 